\title{
Assessment of load on the lumbar spine using two computerised packages and REBA method
}

\author{
TOMASZ MACIEJ TOKARSKI*, DANUTA ROMAN-LIU
}

Central Institute for Labour Protection - National Research Institute, Department of Ergonomics, Warsaw, Poland.

\begin{abstract}
Purpose: The aim of the research was a comparative analysis of the back lumbar load assessment using three methods: two with continuous input data and evaluation procedures based on mathematical relationships (ShiftBack, 3DSSPP) and one method with categorized input data and tabular load estimation procedures (REBA). Methods: For the analysis, work activities and the value of applied force were selected. Among the analyzed 24 work activities were those during which there was a symmetrical load, as well as those during which the applied force or the assumed position of the body caused a lack of load symmetry. Results: The results show that the REBA method differentiates 24 cases of unit load into six levels, while the other two methods provide 24 different values between cases. Cases differing in load even over $100 \%$, when assessed according to the 3DSSPP or ShiftBack do not differ in the assessment of REBA. Differences in the assessment of the same tasks between the results obtained from 3DSSPP and ShiftBack that were above $15 \%$ concern only 7 cases. Conclusions: A comparative analysis of the assessment of the lumbar part of the back using three methods indicates strong correlations between the results of the assessment carried out using 3DSSPP and ShiftBack. Smaller relationships were demonstrated by comparing these two methods with the REBA method.
\end{abstract}

Key words: risk assessment, lower back, comparison of assessment procedures, 3DSSPP, ShiftBack

\section{Introduction}

One of the most important work-related aspects is the excessive load on the musculoskeletal system arising during work-related tasks, which often results in development of musculoskeletal disorders (MSDs) [12]. The back and arms are the regions of the body that are particularly prone to development of MSDs [30]. The percentage share of work-related MSDs in comparison to other conditions in the working population is quite significant and is 59 percent of all problems reported [24]. Not only do work-related MSDs account for the majority of professional pathologies [7], [8], they also often result in absence due to sickness, and even premature retirement [3]. Therefore, the fact that there are places of work where working conditions are inappropriate, the ergonomic principles are neglected, and employees are affected by excessive musculoskeletal load, which all contributes to development of MSDs, is an essential issue that should be considered not only to improve the health and quality of life of employees, but also to prevent financial losses [18].

Development of MSDs is associated with factors characteristic for a specific place of work and often referred to as risk factors [8]. Risk factors are normally classified into three main groups: individual, psychosocial and biomechanical risk factors [11]. The biomechanical factors are most relevant to development of MSDs, and they include posture and force exerted as well as time sequences associated with the two. Posture and force exerted together make up the mechanical load on the lumbar spine, which is the right measure of the risk of developing MSDs in that area [6].

Studies show that the best strategy to prevent MSDs is to maintain the right musculoskeletal load

\footnotetext{
* Corresponding author: Tomasz Maciej Tokarski, Central Institute for Labour Protection - National Research Institute, Department of Ergonomics, ul. Czerniakowska 16, 00-701 Warsaw, Poland. Phone: +48 226233 203, e-mail: tomto@ciop.pl

Received: November 22nd, 2019

Accepted for publication: May 18th, 2020
} 
associated with work-related tasks [4]. For this to be possible, the load needs to be assessed. Therefore, particular importance is attached to the methods where the musculoskeletal load associated with workrelated tasks is assessed. The methods for musculoskeletal load assessment can be discussed in the context of their breakdown into the subjective assessment, direct measurement, and observational methods [8]. The observational methods constitute the most commonly used approach to assessing the musculoskeletal load. The best known and most commonly used observational methods for assessing load are generally based on a simplified human body model reflecting the position of the back and upper and lower limbs. Among the many methods for external load assessment, those most often mentioned are: REBA, RULA [16], KIM (Key Item Method) [26], NIOSH [19], OWAS (Ovako Working Posture Analysis System), OCRA (Occupational Repetitive Actions), SI (Strain Index) or ULRA (Upper Limb Risk Assessment) [21]. These methods allow risks to be identified, changes made to the workstation to be monitored, and research to be carried out on the issues of musculoskeletal load. The assessment is carried out on the basis of variables related to posture and forces exerted. Posture is most often defined by the values of angles in the joints between the body segments involved in a task [22]. However, in some of the methods considered, posture is defined indirectly or qualitatively. On the basis of variables that define posture and force exerted for a single task or number of technical action [27], unit load is calculated and it serves as the basis for further assessment steps which take time sequences into account. In addition number of technical action is one of the main parameters related to the MSDs.

Methods are analysed in the light of various characteristics: type of method, risk factors considered, repeatability and reliability [22]. The results of different MSDs risk assessment methods are also compared [8], [14].

There are both differences and similarities between the methods [22]. The differences mainly relate to the ways of defining the input data to describe posture, force exerted, time sequences and assessment procedures. The assessment procedures consist of simple mathematical relationships or relevant tables. A characteristic shared by all the observational methods is that these assessment methods require the involvement of an expert who performs a time-consuming analysis so that the input data for the method can be defined (variables of posture and force exerted).

The fact that the assessment methods require the involvement of an expert and a time-consuming analy- sis to define the input data is a major impediment to the use of the methods. At the same time, current technological development means that movement can be recorded conveniently by means of markers [10]. When the assessment of musculoskeletal load uses equipment that allows direct collection of data about the position of body parts, it is possible to assume a semi-automatic approach to the assessment of musculoskeletal load and the risk of developing MSDs. Several authors studied the accuracy of the kinematic data provided by a Kinect device [20]. This solution has been used to obtain semi-automated input data for methods such as NIOSH [19], EAWS [15], RULA [16]. The automatised acquisition of input data enables both the accuracy of input data to be increased and more precise assessment procedures to be used. Furthermore, advances in digitisation may also foster development of computerised methods for assessing musculoskeletal load and the risk of developing MSDs. This approach may foster greater accuracy of input data and, as a result, greater precision of the assessment methods. A computerised method of this kind where assessment is based on exact input data, is the recently developed SHIFTRISK method. SHIFTRISK is a computerised method where input data are continuous in form and allow the position of body parts and forces exerted to be defined accurately. Calculations are made on the basis of mathematical relationships, which are embedded in the programme and serve to calculate load in a function of input variables that describe posture, force exerted and time sequences. The method is used to assess the risk of developing MSDs affecting the upper limbs, neck and back (lumbar spine).

The load on the lumbar spine is one of the main measures of workload. The load in this body area is the subject of assessment in such methods as REBA, RULA, NIOSH. A precise assessment of load on the lumbar spine for a specific work-related task, with posture and force exerted taken into account, is possible using computer models such as the 3DSSPP model [28]. The method was developed on the basis of Chaffin's model [5]. However, the result of the assessment carried out using this method only pertains to a specific posture and the corresponding force exerted (unit load). In contrast, the assessment of load and risk of developing MSDs should be comprehensive, pertain to all tasks performed during work, and also include time sequences [23]. Partial assessment, e.g., that of the load on the lumbar spine during a single work task (unit load) being part of a sequence of tasks performed, is an essential element of the assessment of load and risk of developing MSDs. 
The objective of this paper was to provide a comparative analysis of the assessments of unit load on the lumbar spine, where the assessments were carried out using three different methods, differing in the nature of input data and the assessment procedures. Two methods with continuous input data and assessment procedures based on mathematical relationships (ShiftBack, 3DSSPP), and a method with categorised input data and tabular load assessment procedures (REBA) were chosen for the comparison.

\section{Methods}

\subsection{Methods under analysis}

\subsubsection{SHIFTRISK (ShiftBack package)}

The Shiftback package is a constituent part of the SHIFTRISK method and it pertains to the assessment of load on the lumbar spine, which results from a specific posture and exertion of force when performing the tasks that involve the whole body, e.g., when working in a standing position. The SHIFTRISK method is used to assess the risk of developing problems affecting the upper limbs, neck and back (lumbar spine), taking the precise position of the upper limb and precise time characteristics with values of forces into account. The assessment of the load on the lumbar spine is carried out by Shiftback package, using a mathematical relationship to calculate the impact force $\left(F_{\text {back }}\right)$ as a function of the position of the upper limbs, lower limbs and lumbar spine.
In the ShiftBack package, the position of the upper limbs is described by the values of four angles that affects the load on the back and relating to flexion/ extension of the arm $\left(q_{1}\right)$, abduction/adduction of the arm $\left(q_{2}\right)$, rotation of the arm $\left(q_{3}\right)$, flexion at the elbow $\left(q_{4}\right)$ [23]. The position of the lower limbs is determined only with regard to the sagittal plane and the angle of the hip joint $(\Omega)$ and the angle of the knee joint $(\Theta)$ are taken into account (Fig. 1). The position of the lumbar spine is defined by the values of angles in the sagittal $(\alpha)$, coronal $(\beta)$ and transverse $(\gamma)$ plane, and the position of the cervical spine is defined in a similar manner.

In the ShiftBack package which pertains to the assessment of load on the lumbar spine, two types of force are taken into account: the pushing force $\left(F_{\text {push }}\right)$ and the lifting force $\left(F_{\text {lift }}\right)$. Data are entered independently for the left upper limb and the right upper limb.

Thus, in the ShiftBack package, the value of the impact force $\left(F_{\text {back }}\right)$ is calculated as a function of 15 angles (four angles of the upper limb, two angles of the lower limb for the left and right side, and three angles of the back) and the value of the external force (Fig. 2).

The relative load on the lumbar spine for a single task is expressed using an index $\left(R F_{\text {back }}\right)$ is calculated as a value of the impact force $\left(F_{\text {back }}\right)$ in relation to the maximum force $\left(F_{\max }\right)$ corresponding to the lumbar spine strength $\left(F_{\text {backMax }}\right)$. The spine is capable of transferring very heavy loads that range from $4.5 \mathrm{kN}$ (about $450 \mathrm{~kg}$ ) for the third cervical vertebra (C3) up to $8.3 \mathrm{kN}$ (about $830 \mathrm{~kg}$ ) for the fourth lumbar vertebra (L4) [2]. It was therefore assumed that $F_{\text {backMax }}$ is a fixed value of $8.3 \mathrm{kN}$. The $R F_{\text {back }}$ force is a load index to be compared with the indices obtained from other methods.
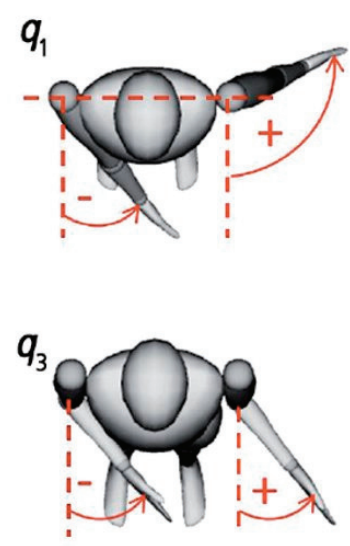
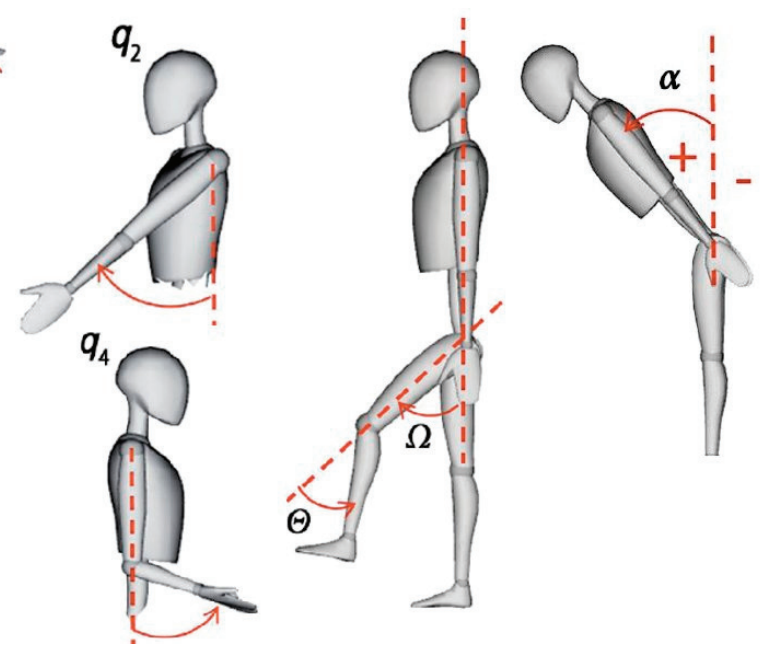

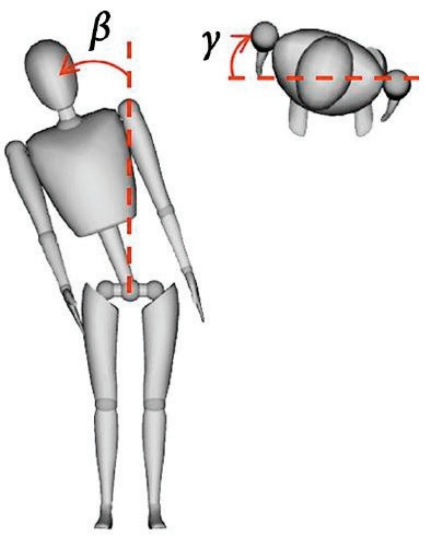

Fig. 1. Manner of defining the angles of the positions of the upper and lower limbs that will serve as input data for load assessment 


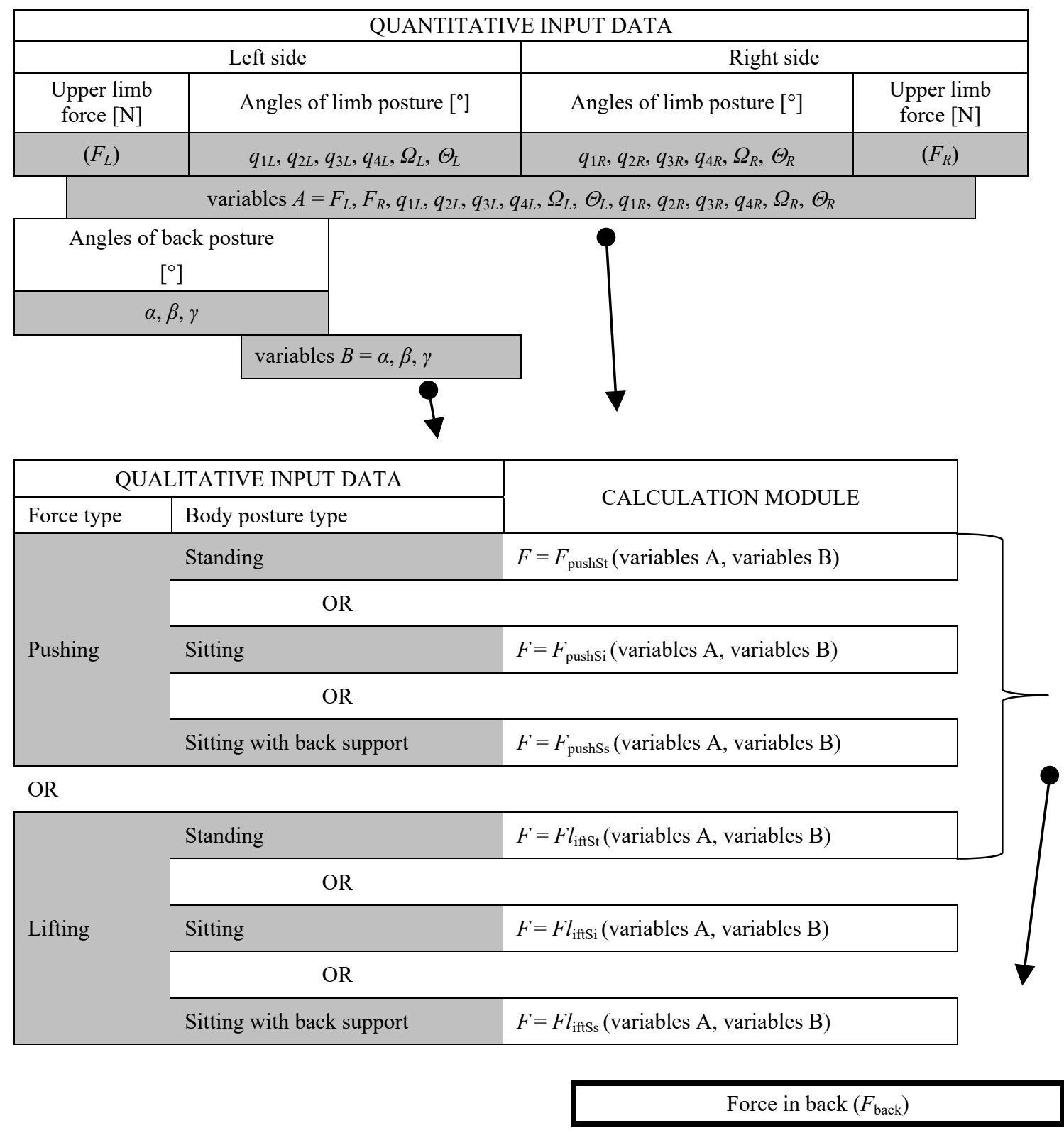

Fig. 2. Diagram showing the ShiftBack package calculations including input data

\subsubsection{Static Strength Prediction Program (3DSSPP)}

In the 3DSSPP (Static Strength Prediction Program) method, the calculation of musculoskeletal load is based on a simplified model of the whole human body, which consists of twelve segments representing body parts (upper torso and the head, lower torso, arms, forearms and hands, thighs, lower legs, feet). The mathematical description of the model is developed using the Newton -Euler equations and allows static analysis of two- and three-dimensional problems [5].

Anthropometric parameters describing an employee (data for different populations can be used), posture during work (defined by the provided values of angles in the joints) and the value of the external force constitute the input data for the programme.

The individual angles of the positions of body parts are defined in reference to two planes only. In the 3DSSPP method, the position of the upper limb is defined by values of the following angles: arm position angles in the coronal (Va) and transverse (Ha) plane, and forearm angles in the coronal (Vf) and transverse (Hf) plane (Fig. 3).

Two planes, the sagittal and the transverse plane, were also taken into account when defining the position of the lower limbs. The angles in the hip and the knee joints are determined: the thigh position angle $(\mathrm{Vu}, \mathrm{Hu})$ and the lower leg position angle (Vl, Hl). The force exerted is entered as a value and type of force. Lifting, 

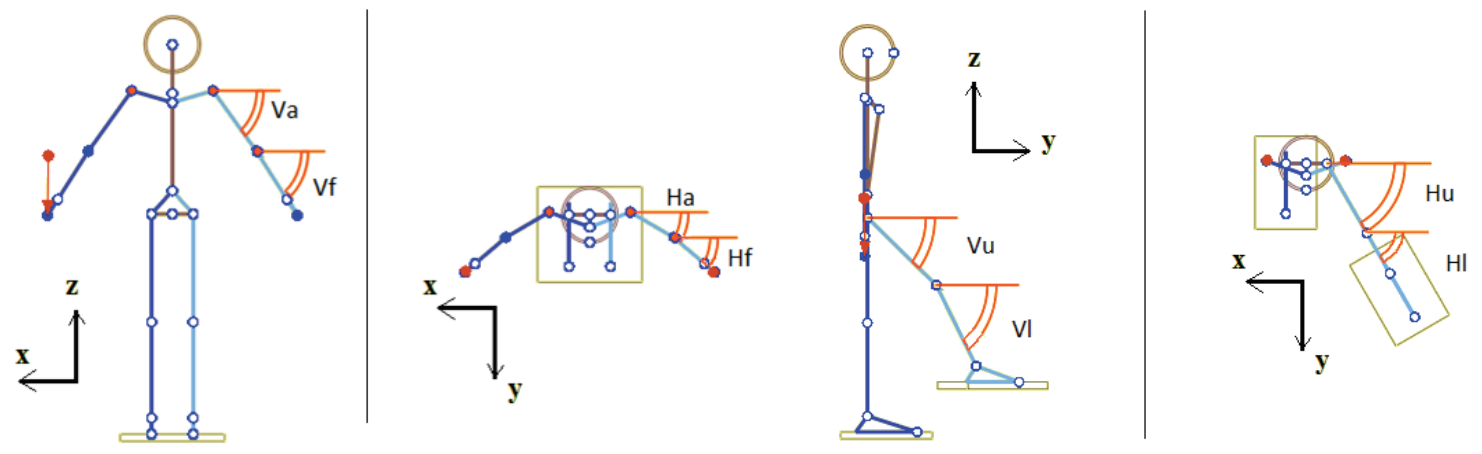

Fig. 3. Defining the upper and lower limb position in the 3DSSPP method

pushing and pulling forces are taken into account When loads are handled manually, the calculations include the absolute force value applied to the middle part of the palm. The assessment carried out in accordance with the 3DSSPP method resulted in the value of a compressive force in the lumbar spine (L4/L5 disc), which is to be compared with results of the other two methods.

\subsubsection{REBA}

In order to identify the position of body parts, the REBA method uses a range of values of the angle at the joint rather than a value of the angle. The position so defined is assigned a code which depends on the limit values of the range of the angle considered. The first step defines posture only in the sagittal plane. If a posture requires that the position in other planes must also be taken into account, e.g., when there is abduction/adduction or pronation/supination, the value of the assigned code is modified. The assessment made using this method is broken down into two groups. Group A assessment covers the assessment of the back on the basis of the position of the back, neck and legs. Group B assessment concerns the upper limbs. To combine the codes reflecting the position of the individual body segments into a single assessment category, relevant tables are used. For group A, the position category determined on the basis of codes that represent the position of the back, neck and legs is combined with the code representing the force in the next assessment step.

The position of the back is defined in reference to the lumbar and cervical part. For any head inclination angle, codes 1 or 2 are assigned depending on whether the inclination of the head is between $0^{\circ}$ and $20^{\circ}$ or more than $20^{\circ}$. If the neck is twisted, or when any lateral inclination of the head occurs, the code is increased by 1 . For the back, codes from 1 to 4 may be assumed depending on the back inclination angle. The limit values for the subsequent codes are the values of the back inclination angle of $0^{\circ}, 20^{\circ}$ and $60^{\circ}$ respectively. The back position code value is increased by 1 both when the back is twisted and when it is inclined laterally. For the position of the legs, a code from 1 to 4 is assumed, depending on body weight distribution and values of the angle at the knee joint.

The code representing the force is determined according to the value of the force and may be 0 (less than $5 \mathrm{~kg}$ ), 1 (a force of 5-10 kg) or 2 (a force of more than $10 \mathrm{~kg}$ ). When the force is exerted in a rapid manner, the previously assigned code is additionally increased by 1 . The relevant table combines the neck, back and leg codes to obtain an A position category of the REBA method.

The comparison of the results of assessment, which is the subject of this article, only pertains to group A assessment, and the result of group A assessment (load category A) will be the load index obtained from the REBA method.

\subsection{Comparative load assessment procedure using 3DSSPP, SHIFTISK and REBA methods}

Regardless of the type of task performed, the methods for external load assessment express load in a function of input parameters. In these methods, load pertains to a single task and a series of sequences of the workrelated tasks performed. To satisfy the research objective presented in the paper, only the assessment of the load arising due to a single task will be considered, which means that the values of angles in the joints, defining posture and value of the force exerted, will be used as input data to assess the load.

The 3DSSPP, SHIFTISK and REBA methods differ in both the manner of entering input data and the procedure for calculating load indices. Therefore, the assessment is carried out using procedures that differ according to method. However, for the purposes of the 
analysis, the same model for the initial definition of input data was assumed for all the methods. It was assumed that the position of the upper limb is defined by the values of four angles, the position of the back is defined by the values of three angles in the lumbar spine and three angles in the cervical spine, and the position of the legs is defined by two angles.

The analysis concerned the values of load obtained as a result of assessment using the 3DSSPP, ShiftBack and REBA methods, as well as the relationships between the results obtained from the three methods.

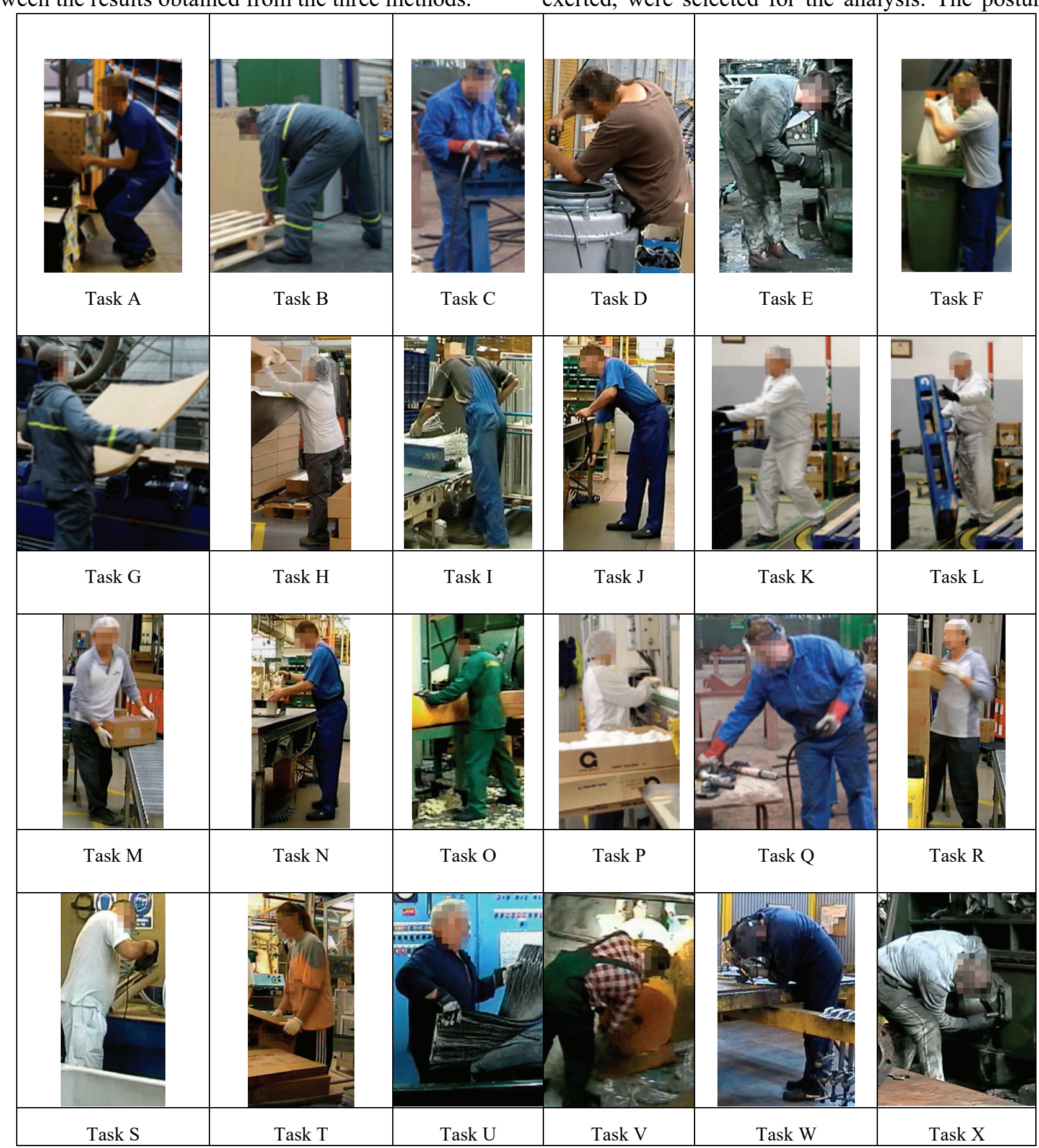

Fig. 4. Postures of employees during various work-related tasks, assessed and analysed for load 
Table 1. Analysed work-related tasks with their assigned values of force exerted and angles at the upper limb joints (* - pushing force, in other cases lifting force)

\begin{tabular}{|c|c|c|c|c|c|c|c|c|c|c|c|}
\hline \multicolumn{2}{|c|}{ Posture } & $\alpha\left[^{\circ}\right]$ & $\beta\left[^{\circ}\right]$ & $\gamma\left[^{\circ}\right]$ & $q_{1}\left[^{\circ}\right]$ & $q_{2}\left[{ }^{\circ}\right]$ & $q_{3}\left[{ }^{\circ}\right]$ & $q_{4}\left[^{\circ}\right]$ & $\Omega\left[^{\circ}\right]$ & $\Theta\left[^{\circ}\right]$ & $F[\mathrm{~N}]$ \\
\hline \multirow{2}{*}{ A } & left & 20 & 0 & 0 & 0 & 20 & 0 & 90 & 40 & 90 & 80 \\
\hline & right & 20 & 0 & 0 & 0 & 20 & 0 & 90 & 40 & 90 & 80 \\
\hline \multirow{2}{*}{ B } & left & 90 & 0 & 0 & -10 & 80 & 0 & 0 & 20 & 20 & 60 \\
\hline & right & 90 & 10 & 0 & -10 & 80 & 0 & 0 & 20 & 20 & 60 \\
\hline \multirow{2}{*}{$\mathrm{C}$} & left & 10 & 0 & 0 & 20 & 20 & -20 & 90 & 0 & 0 & 0 \\
\hline & right & 10 & 0 & 0 & 20 & 20 & -20 & 90 & 20 & 20 & 40 \\
\hline \multirow{2}{*}{$\mathrm{D}$} & left & 10 & 0 & 0 & 10 & 50 & -10 & 90 & 0 & 0 & 0 \\
\hline & right & 10 & 0 & 0 & 30 & 110 & -100 & 110 & 0 & 0 & 80 \\
\hline \multirow{2}{*}{$\mathrm{E}$} & left & 30 & 0 & 0 & 20 & 30 & -30 & 10 & 10 & 10 & 110 \\
\hline & right & 30 & 0 & 0 & 20 & 40 & -20 & 80 & 10 & 10 & 110 \\
\hline \multirow{2}{*}{$\mathrm{F}$} & left & 0 & 0 & 0 & 20 & 60 & -40 & 90 & 0 & 0 & 100 \\
\hline & right & 0 & 0 & 0 & 20 & 60 & -40 & 90 & 0 & 0 & 100 \\
\hline \multirow{2}{*}{ G } & left & 0 & 0 & 0 & 80 & 40 & -40 & 50 & 0 & 0 & 100 \\
\hline & right & 0 & 0 & 0 & 80 & 40 & -40 & 50 & 0 & 0 & 100 \\
\hline \multirow{2}{*}{$\mathrm{H}$} & left & 0 & 0 & 0 & 0 & 90 & -20 & 30 & 0 & 0 & $5^{*}$ \\
\hline & right & 0 & 0 & 0 & 0 & 90 & -20 & 30 & 0 & 0 & $5^{*}$ \\
\hline \multirow{2}{*}{ I } & left & 30 & 0 & 0 & 20 & 50 & -30 & 20 & 0 & 0 & 5 \\
\hline & right & 30 & 0 & 0 & 150 & 20 & -150 & 110 & 20 & 0 & 0 \\
\hline \multirow{2}{*}{$\mathrm{J}$} & left & 50 & 0 & 0 & 40 & 40 & -80 & 90 & 0 & 0 & 70 \\
\hline & right & 50 & 0 & 10 & -10 & 60 & 0 & 0 & 10 & 0 & 70 \\
\hline \multirow{2}{*}{ K } & left & 10 & 0 & 0 & 10 & 70 & -10 & 10 & 30 & 40 & $130^{*}$ \\
\hline & right & 10 & 0 & 0 & 10 & 50 & -10 & 40 & 20 & 0 & $130^{*}$ \\
\hline \multirow{2}{*}{$\mathrm{L}$} & left & 0 & 0 & 0 & 20 & 90 & -20 & 10 & 0 & 0 & $130^{*}$ \\
\hline & right & 0 & 0 & 0 & -10 & 50 & 0 & 40 & 0 & 0 & $130^{*}$ \\
\hline \multirow{2}{*}{$\mathrm{M}$} & left & 20 & 0 & 0 & 0 & 30 & 0 & 20 & 20 & 20 & 40 \\
\hline & right & 20 & 10 & 0 & 0 & 30 & 0 & 20 & 20 & 20 & 40 \\
\hline \multirow{2}{*}{$\mathrm{N}$} & left & 10 & 0 & 0 & 20 & 50 & -20 & 70 & 0 & 0 & 30 \\
\hline & right & 10 & 0 & 0 & 20 & 50 & 10 & 90 & 0 & 0 & 30 \\
\hline \multirow{2}{*}{$\mathrm{O}$} & left & 0 & 0 & 0 & 40 & 40 & -60 & 70 & 0 & 0 & 0 \\
\hline & right & 0 & 0 & 0 & 40 & 40 & -10 & 40 & 0 & 0 & 0 \\
\hline \multirow{2}{*}{$\mathrm{P}$} & left & 0 & 0 & 0 & 0 & 50 & -20 & 60 & 0 & 0 & 0 \\
\hline & right & 0 & 0 & 0 & 0 & 50 & -20 & 60 & 0 & 0 & 3 \\
\hline \multirow{2}{*}{$\mathrm{Q}$} & left & 20 & 0 & 0 & 30 & 10 & -70 & 70 & 0 & 0 & 40 \\
\hline & right & 20 & 10 & 0 & 40 & 70 & 0 & 10 & 0 & 0 & 0 \\
\hline \multirow{2}{*}{$\mathrm{R}$} & left & 0 & 0 & 0 & 10 & 10 & 0 & 130 & 0 & 0 & 0 \\
\hline & right & 0 & 0 & 0 & 10 & 10 & 0 & 130 & 0 & 0 & 10 \\
\hline & left & 10 & 0 & 0 & 20 & 20 & 0 & 10 & 0 & 0 & 10 \\
\hline S & right & 10 & 0 & 0 & 80 & 40 & -120 & 110 & 0 & 0 & 90 \\
\hline & left & 0 & 0 & 0 & 0 & 0 & 0 & 20 & 0 & 0 & 20 \\
\hline $\mathrm{T}$ & right & 0 & 0 & 0 & 0 & 0 & 0 & 20 & 0 & 0 & 20 \\
\hline & left & 0 & 0 & 0 & 20 & 10 & 0 & 120 & 0 & 0 & 30 \\
\hline 0 & right & 0 & 0 & 0 & 90 & 80 & 0 & 100 & 0 & 0 & 30 \\
\hline & left & 60 & 0 & 0 & 30 & 100 & 0 & 0 & 0 & 0 & 0 \\
\hline V & right & 60 & 0 & 10 & 80 & 20 & -120 & 110 & 20 & 0 & 50 \\
\hline & left & 30 & 0 & 0 & 30 & 110 & -100 & 110 & 0 & 0 & 70 \\
\hline W & right & 30 & 0 & 0 & 10 & 40 & -70 & 100 & 0 & 0 & 70 \\
\hline $\mathrm{y}$ & left & 70 & 0 & 10 & 30 & 110 & -100 & 110 & 0 & 0 & 110 \\
\hline$x$ & right & 70 & 0 & 0 & -10 & 70 & -20 & 90 & 40 & 40 & 110 \\
\hline
\end{tabular}

Note: $\alpha$ - angle of back flexion, $\beta$ - angle of back lateral bending, $\gamma$ - angle of back rotation, $q_{1}-$ angle of arm flexion/extension, $q_{2}$ - angle of arm abduction/adduction, $q_{3}$ - angle of arm rotation, $q_{4}$ - angle of flexion at the elbow, $\Omega$ - angle at the hip joint; $\Theta$ - angle at the knee joint; $F$ - force exerted. 
are shown in Fig. 4, whereas the values of the angles defining the position of the upper limbs, back and legs, and the values of the force exerted, are shown in Table 1. Postures were defined in accordance with a general principle, also adopted by the SHIFTRISK method. According to that principle, the values of all angles in the natural posture (upright, standing) are zero. Designated values of the angles were determined on the basis of observations and videos recorded during observation of work activities. The load assessment was carried out by specialists in the field of ergonomics with many years of experience in the assessment of load using methods such as REBA, RULA, NIOSH, OCRA and measurements using muscle forces, electromyography, goniometers.

\subsection{Results of the analysis}

In the calculation using the ShiftBack package, the values of forces and angles shown in Table 1 were used as input data. For the calculation using the 3DSSPP method, the values of the angles needed to be transposed as the 3DSSPP method assumes a connotation of the values of the position angles that is different from the common approach. In the REBA method, values of particular angles are replaced by values of codes. The basic codes corresponding to the angles of changes in the position in the sagittal plane, and the supporting codes that refer to the position in the coronal plane, are taken into account. The codes representing load on the back, neck and legs, and the codes representing the external force, served as the basis for calculating overall load in group A.

Table 2. Results of the assessment of load on the lumbar spine obtained from the 3DSSPP, ShiftBack and REBA methods

\begin{tabular}{|l|c|c|c|}
\hline \multicolumn{1}{|c|}{$\mathbf{1}$} & $\begin{array}{c}\text { ShiftBack } \\
{[\mathrm{N}]}\end{array}$ & $\begin{array}{c}\text { 3DSSPP } \\
{[\mathrm{N}]}\end{array}$ & $\begin{array}{c}\text { Load } \\
\text { assessment A }\end{array}$ \\
\hline Posture A & 2637.8 & 2579 & $\mathbf{4}$ \\
\hline Posture B & 3900.3 & 3718 & 7 \\
\hline Posture C & 1512.0 & 1300 & 9 \\
\hline Posture D & 1365.9 & 1515 & 7 \\
\hline Posture E & 3397.5 & 3078 & 8 \\
\hline Posture F & 1722.6 & 1951 & 6 \\
\hline Posture G & 769.0 & 1427 & 5 \\
\hline Posture H & 827.8 & 788 & 4 \\
\hline Posture I & 1920.0 & 1742 & 7 \\
\hline Posture J & 4217.6 & 3148 & 9 \\
\hline
\end{tabular}

\begin{tabular}{|l|c|c|c|}
\hline \multicolumn{1}{|c|}{$\mathbf{1}$} & $\mathbf{2}$ & $\mathbf{3}$ & $\mathbf{4}$ \\
\hline Posture K & 3503.9 & 3458 & 7 \\
\hline Posture L & 2956.3 & 3296 & 7 \\
\hline Posture M & 2380.4 & 1891 & 7 \\
\hline Posture N & 1506.1 & 1571 & 5 \\
\hline Posture O & 595.6 & 548 & 4 \\
\hline Posture P & 749.2 & 643 & 4 \\
\hline Posture Q & 1738.4 & 1636 & 7 \\
\hline Posture R & 627.1 & 458 & 4 \\
\hline Posture S & 1050.6 & 1493 & 6 \\
\hline Posture T & 582.9 & 416 & 4 \\
\hline Posture U & 874.4 & 695 & 4 \\
\hline Posture V & 2873.8 & 2853 & 8 \\
\hline Posture W & 2399.6 & 2563 & 9 \\
\hline Posture X & 4784.8 & 4758 & 11 \\
\hline
\end{tabular}

Table 2 shows the results of calculation of the unit load arising from posture and force exerted, obtained using the three methods for 24 different tasks. Figure 5 shows variation in the values of load calculated according to the Shiftback and 3DSSPP methods for the same values of the load category determined as per the REBA method. Figure 6 shows the relationship between the values of load calculated using the 3DSSPP method and the Shiftback and REBA methods.

In Figure 7, values of load of ShiftBack and 3DSSPP assigned to specific codes obtained according to REBA method are presented. The differences in load assessment done with ShiftBack or 3DSSPP for cases that are not differentiated with REBA method are shown.

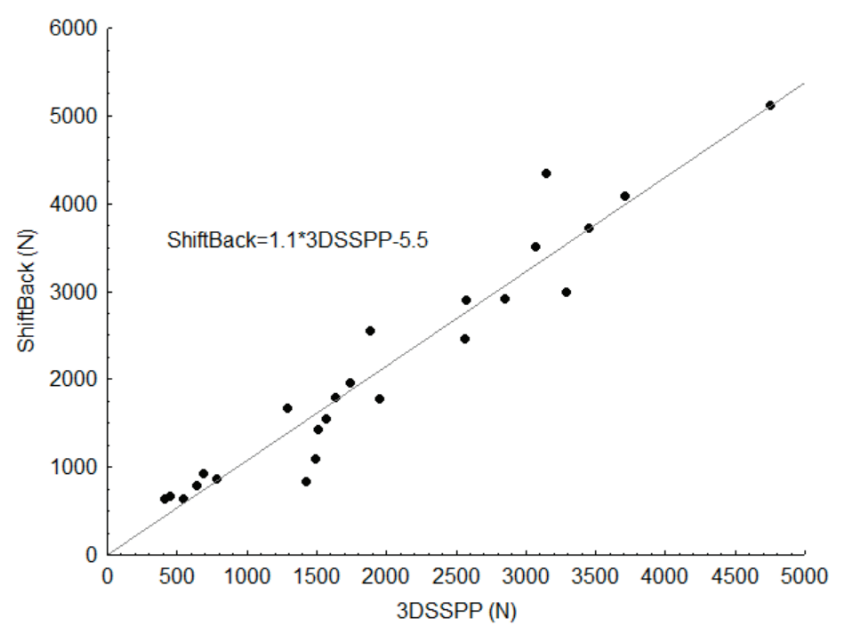

Fig. 5. Values of load on the lumbar spine for specific tasks, calculated using the Shiftback and 3DSSPP methods and ranked according to the assessment category obtained from the REBA method 


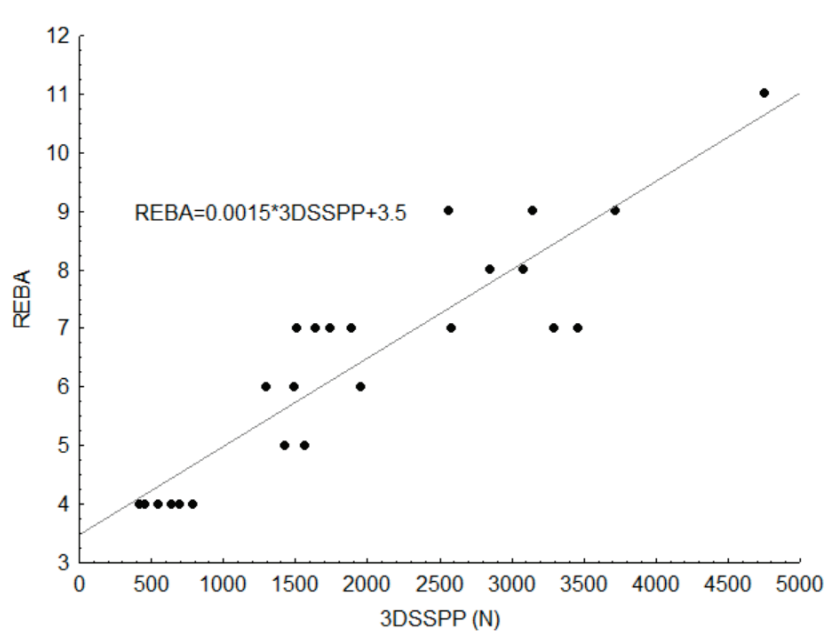

Fig. 6. Relationships between the results of load assessment obtained using the Shiftback method and the 3DSSPP method, and using the REBA method and the 3DSSPP method

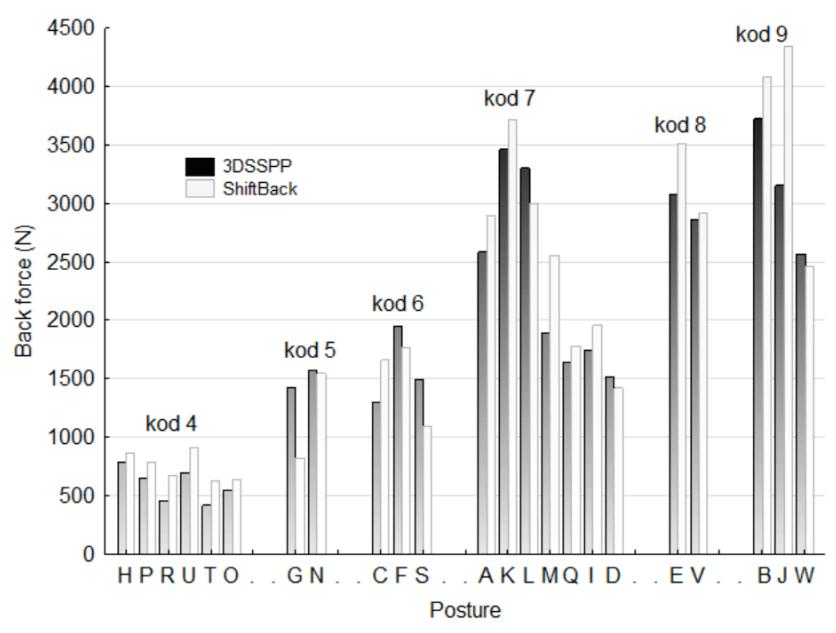

Fig. 7. Differences in load assessment using ShiftBack or 3DSSPP for cases that are not differentiated with REBA method

\section{Discussion}

Many publications highlight the importance the unit load, made up of posture and force exerted during work, has for the development of MSDs. The main way to prevent the development of an MSDs is to ensure that an employee's workload is appropriate. To assess whether it is appropriate, it is necessary to select the right assessment method and carry out a quantitative analysis of the risk associated with the work tasks. The purpose of the tests presented in this paper was to analyse the differences and similarities between the assessments of load on the lumbar spine, performed using different assessment procedures and varying accuracy of input data. For the comparison, three procedures were selected, each of them being a constituent part of the ShiftBack package being a part of the SHIFTRISK method, REBA and 3DSSPP methods [28].

The 3DSSPP method is a commonly known and used method of load assessment. The model used for the 3DSSPP calculations is a relatively precise model, compared to the models adopted in other methods, and it can serve as a reference for other methods. The weakness of the 3DSSPP method is that it does not allow for overall assessment along with the risk assessment, i.e., assessment that includes a sequence of work tasks with time sequences. This is possible with the SHIFTRISK method, where the load on the back is assessed on the basis of the ShiftBack package. This method allows both unit assessment, i.e., assessment of unit load related to specific postures along with force exerted, and assessment of overall load arising during a sequence of tasks, taking the relevant time sequences into account.

In analysis presented in this paper, 24 different postures with forces exerted were analysed. The analysed work tasks included those where a symmetrical load occurred, and those where the force exerted or the posture assumed resulted in the lack of symmetry of the load. The results of the comparative analysis indicate strong relationships between the assessment according to the ShiftBack package and the assessment using 3DSSPP. The comparison of the REBA method and the 3DSSPP method showed a weaker relationship. The results show that the REBA method differentiates the 24 cases of unit load into six levels, whereas the two other methods deliver 24 values differentiated among the cases. Therefore, the cases that, according to the assessment by the 3DSSPP and ShiftBack methods, differ in load even by more than 100 percent, are not different when assessed by the REBA method. In the case of the REBA, the range of angles that define the position of the back is divided into 7 areas only. This means that the accuracy of input data is lower.

The differences between the results obtained from the 3DSSPP and the ShiftBack methods are relatively small. Differences of more than 15 percent were found in 7 cases. Differences in assessment of the same tasks may be due to differences in the manner of identifying the variables that describe the angular positions of body parts and force, and differences in the manner of assessing load [29]. The discreet nature of the REBA method and the more continuous nature of the ShiftBack and 3DSSPP methods may be the main source of the differences. Since the input data in the ShiftBack package and in the 3DSSPP were of the same nature, it should be assumed that the minor differences in the load assessed according to the 
ShiftBack package and according to the 3DSSPP method are mainly due to differences in the calculation procedure. On the other hand, the differences between these two methods and the REBA are linked to both the accuracy of the input data and the type of calculation procedure. Differences in the results of load assessment obtained using the 3DSSPP and the ShiftBack may also be due to anthropometric dimensions of the subjects. The 3DSSPP takes account of the centile of population for which the calculations are carried out. In the ShiftBack, anthropometric measurements are not taken into account.

The study showed a relationship between methods for assessing musculoskeletal load. ShiftBack itself and the results of the presented analysis give scientists the basis for further work on the back load and tools for its assessment. The results of the study proved the usefulness of the ShiftBack procedure. This is an innovative tool for ergonomics practitioners, therapists, employers and employees need the effective assessment methods available to prevent MSDs. Many methods of risk assessment have been developed [3], [7], [13], [17], [22]. All of the available risk assessment methods require input data and include specific assessment procedures. Methods for assessing the load and risk of developing MSDs differ in how they define the parameters related to posture, force and time sequences, how they assess the load and, in some cases, how they determine the amount of load-related risk [22]. Different methods adopt different models of the human body. The choice of an appropriate method for assessing the workload requires a compromise between accuracy and feasibility (i.e., in time and cost terms). Usually, the methods based on subjective assessment are used fairly widely and involve a large group of employees; however, due to the subjectivity and the categorised assessment, these methods have limited accuracy [1]. At the same time, the assessment of musculoskeletal load may differ when different methods are used [26], [8]. This may make the choice of an appropriate tool difficult [22]. None of the methods is exhaustive with regard to all aspects of load assessment. Often, using more than one assessment method may help to establish priorities for ergonomic intervention and provide a more accurate assessment. On the other hand, using more than one method can quickly lead to unacceptably high assessment-related costs [8]. It would be most appropriate to use a method that allows the load and the risk of developing MSDs to be assessed in the light of all the work tasks an employee performs during their working day. In the light of the technological developments offering automatised measurements of the position of body parts
[20] and computerised calculation procedures, this method could be of broad interest. A dramatic reduction should be expected in the costs of using the accurate posture recording methods, e.g., inertia sensors or automated body position tracking without markers [9] together with a procedures that would allow for precise assessment of load and risk for MSDs.

\section{Conclusion}

A comparative analysis of the assessments of work task related load on the lumbar spine, where the assessments were carried out using three different methods, indicates strong correlations between the results obtained from the assessment methods that used continuous input data and assessment is based on mathematical relationships. More minor relationships were shown when these two methods were compared with a method that used categorised input data and assessment procedures. These methods deliver a more differentiated assessment than the method with the categorised system of input data and assessment.

Assessment of work/task related load on the on the lumbar spine in different positions and at different loads showed that the ShiftBack and 3DSSPP methods of analysis are more accurate and detailed than the REBA method, which treated this topic more generally. Results of this study confirm the importance that the nature of input data and assessment procedures has for the musculoskeletal load assessment. The study showed that the ShiftBack method is suitable for load assessment and further scientific research to reduce back load. It is also an innovative tool for professionals involved in the prevention of MSDs.

\section{Acknowledgement}

This paper has been based on the results of a research task carried out within the scope of the second stage of the National Programme "Improvement of safety and working conditions" partly supported in 2017-2019 - within the scope of state services - by the Ministry of Labour and Social Policy. The Central Institute for Labour Protection e National Research Institute is the Programme's co-ordinator.

\section{References}

[1] Balogh I., Ørbaek P., Ohlsson K., Nordander C., Unge J., Winkel J., Hansson G.A., Malmö Shoulder/Neck Study Group. Self-assessed and directly measured occupational physical activities - influence of musculoskeletal complaints, age and gender, Appl. Ergon., 2004, 35, 49-56. 
[2] Borkowski P., Biomechanical analysis and design of a new type of artificial intervertebral disc (in Polish Biomechaniczna analiza i projektowanie nowego typu sztucznego krązka międzykręgowego), doctoral thesis, 2006.

[3] Bulduk E.Ö., Bulduk S., SüREn T., Ovali F., Assessing exposure to risk factors for work-related musculoskeletal disorders using Quick Exposure Check (QEC) in taxi drivers, Int. J. Ind. Ergon., 2014, 44 (6), 817-820.

[4] BURDORF A., The role of assessment of biomechanical exposure at the workplace in the prevention of musculoskeletal disorders, Scand. J. Work Environ. Health, 2010, 36, 1-2.

[5] Chaffin D.B., Andersson G., Martin J., Occupational Biomechanics, fourth ed., Wiley, New York, 2006.

[6] CHAFFIN D.B., The evolving role of biomechanics in prevention of overexertion injuries, Ergonomics, 2009, 52, 3-14.

[7] Chander D.S., Cavatorta M.P., An observational method for postural ergonomic risk assessment (PERA), Int. J. Ind. Ergon, 2017, 57, 32-41.

[8] Chiasson M.È., Imbeau D., Aubry K., Delisle A., Comparing the results of eight methods used to evaluate risk factors associated with musculoskeletal disorders, Int. J. Ind. Ergon, 2012, 42 (5), 478-488.

[9] DuTTA T., Evaluation of the KinectTM sensor for 3-D kinematic measurement in the workplace, Appl. Ergon., 2012, 43 (4), 645-649.

[10] FEDEROLF P.A., A novel approach to study human posture control: "principal movements" obtained from a principal component analysis of kinematic marker data, J. Biomech., 2016, 49 (3), 364 370, http://dx.doi.org/10.1016/j.jbiomech.2015.12.030.

[11] Ferguson S.A., Allread W.G., Burr D.L., Heaney C., MARRAS W.S., Biomechanical, psychosocial and individual risk factors predicting low back functional impairment among furniture distribution employees, Clin. Biomech. (Bristol, Avon), 2012, 27 (2), 117-123.

[12] Griffith L.E., Shannon H.S., Wells R.P., Walter S.D., Cole D.C., Côté P., Frank J., HogG-Johnson S., LANGlois L.E., Individual participant data meta-analysis of mechanical workplace risk factors and low back pain, Am. J. Public Health, 2012, 102, 309-318.

[13] Hellig T., Mertens A., Brandl C., The interaction effect of working postures on muscle activity and subjective discomfort during static working postures and its correlation with OWAS, Int. J. Ind. Ergon., 2018, 68, 25-33.

[14] Kong Y.K., LeE S.Y., LeE K.S., KIM D.M., Comparisons of ergonomic evaluation tools (ALLA, RULA, REBA and OWAS) for farm work, Int. J. Occup. Saf. Ergon., 2018, 24 (2), 218-223.

[15] Kruger J., NGuYen T.D., Automated vision-based live ergonomics analysis in assembly operations, CIRP Ann.-Manuf. Techn. 2015, 64, 9-12.

[16] Manghisi V.M., Uva A.E., Fiorentino M., Bevilacqua V., Trotta G.F., Monno G., Real time RULA assessment using Kinect v2 sensor, Appl. Ergon., 2017, 65, 481-491.
[17] Maurice P., Padois V., Measson Y., Bidaud P., Humanoriented design of colla-borative robots, Int. J. Ind. Ergon., 2017, 57, 88-102.

[18] Отто A., Scholl A., Incorporating ergonomic risks into assembly line balancing, European Journal of Operational Research, 2011, 212 (2), 277-286.

[19] Patrizi A., Pennestr E., Valentini P.P., Comparison between low-cost markerless and high-end marker-based motion capture systems for the computeraided assessment of working ergonomics, Ergonomics, 2015, 1-8.

[20] Plantard P., Auvinet E., Pierres A.-S.L., Multon F., Pose estimation with a kinect for ergonomic studies: evaluation of the accuracy using a virtual mannequin, Sensors, $2015,15,1785-1803$.

[21] Roman-Liu D., Groborz A., ToKarski T., Comparison of risk assessment procedures used in OCRA and ULRA methods, Ergonomics, 2013, 56 (10), 1584-1598.

[22] Roman-Liu D., Comparison of concepts in easy-to-use methods for MSD risk assessment, App. Ergon., 2014, 45 (3), $420-427$.

[23] Roman-Liu D., External load and the reaction of the musculoskeletal system-A conceptual model of the interaction, Int. J. Ind. Ergonom., 2013, 43(4), 356-362.

[24] Schneider E., IRASTORZA X., COPSEY S., OSH in Figures: Work-related Musculoskeletal Disorders in the EU d Facts and Figures, 2010, http://dx.doi.org /10.2802/10952.

[25] Sielholz P., Bao S., Howard N., Silverstein B., Fan J., SMith C., Salazar C., Reliability and validity assessment of the hand activity level threshold limit value and strain index using expert ratings of mono-task jobs, J. Occup. Environ. Hyg., 2008, 5 (4), 250-257.

[26] Steinberg U., Caffier G., Liebers F., Assessment of manual material handling based on key indicators - german guidelines, [in:] W. Karwowski (eds.), Handbook of standards in ergonomics and human factors, Lawrenz Erlbaum Associates, Mahwah, New Jersey, 2006, 319-338.

[27] Taborri J., Bordignon M., Marcolin F., Bertoz A., DOnATI M., Rossi S., On the OCRA Measurement: Automatic Computation of the Dynamic Technical Action Frequency Factor, Sensors, 2020, 20, 1643, 10.3390/s20061643.

[28] University of Michigan Center for Ergonomics, 3D Static Strength Prediction Program User's Manual, 2014.

[20] VAN DER BeEK A.J., Mathiassen S.E., Windhorst J., BURDORF A., An evaluation of methods assessing the physical demands of manual lifting in scaffolding, App. Ergon., 2005, 36, 213-222.

[30] Widanarko B., Legg S., Stevenson M., Devereux J., Eng A., Mannetje A.T., Cheng S., Douwes J., Ellison-Loschmann L., McLean D., Pearce N., Prevalence of musculoskeletal symptoms in relation to gender, age, and occupational/industrial group, Int. J. Ind. Ergon., 2011, 41 (5), 561-572. 